\title{
Psicose induzida por drogas recreativas: uma revisão de literatura
}

\author{
Recreational drug-induced psychosis: a literature review \\ Psicosis inducida por drogas recreativas: revisión de la literatura
}

Recebido: 28/01/2021 | Revisado: 04/02/2021 | Aceito: 05/02/2021 | Publicado: 13/02/2021

\author{
Daniel Nobrega Medeiros \\ ORCID: https://orcid.org/0000-0003-3604-7380 \\ Universidade de Vassouras, Brasil \\ E-mail: danielmedeiros.medeiros@gmail.com \\ Juliana Fernandes de Souza Ribeiro \\ ORCID: https://orcid.org/0000-0001-8211-7330 \\ Universidade de Vassouras, Brasil \\ E-mail: julianafdesouza@yahoo.com.br \\ Larissa Alexsandra da Silva Neto Trajano \\ ORCID: https://orcid.org/0000-0002-2600-9770 \\ Universidade de Vassouras, Brasil \\ E-mail: larissa.alexsandra@hotmail.com
}

\begin{abstract}
Resumo
Psicose é um termo amplo que caracteriza uma série de distúrbios que têm em comum a produção dos chamados sintomas psicóticos, principalmente delírios e alucinações. Os transtornos psicóticos podem ser divididos em primários e secundários, incluindo-se nesta última categoria os transtornos psicóticos induzidos por substâncias, dentre as quais se destacam as drogas recreativas. O objetivo desta revisão foi analisar as principais características fisiopatológicas, sintomáticas, diagnósticas e terapêuticas dos transtornos psicóticos relacionados ao uso de drogas recreativas. Foi realizada uma busca por trabalhos prévios nas plataformas SciELO, Lilacs e PubMed e um total de 27 artigos científicos foram incluídos após a aplicação de critérios de inclusão e exclusão. Através dos estudos analisados foi observado que as drogas mais frequentemente associadas com transtornos psicóticos foram cannabis, anfetaminas, o álcool e a cocaína, sendo que os delírios, especialmente persecutórios, e as alucinações auditivas foram os principais sintomas relatados. Embora a fisiopatologia destes distúrbios não esteja completamente esclarecida, há evidências de que o uso de drogas ilícitas sejam um fator de risco para o desenvolvimento de eventos psicóticos, desta forma, o conhecimento dos fatores envolvidos nessa relação é importante na prática clínica. Em conclusão, é importante que os profissionais de saúde saibam que o uso de drogas recreativas pode levar ao desenvolvimento de psicose e que saibam avaliar a melhor forma de abordar esses pacientes para que possam oferecer o melhor suporte possível.
\end{abstract}

Palavras-chave: Psicose; Transtornos psicóticos; Substâncias; Drogas recreativas.

\begin{abstract}
Psychosis is a broad term that characterizes a series of disorders that have in common the production of so-called psychotic symptoms, mainly delusions and hallucinations. Psychotic disorders can be divided into primary and secondary, including substance-induced psychotic disorders in this last category, among which recreational drugs stand out. The aim of this review was to analyze the main pathophysiological, symptomatic, diagnostic and therapeutic characteristics of psychotic disorders related to the use of recreational drugs. A search for previous works was carried out on the SciELO, Lilacs and PubMed platforms and a total of 27 scientific articles were included after application of inclusion and exclusion criteria. Through the studies analyzed, it was observed that the drugs most often associated with psychotic disorders were cannabis, amphetamines, alcohol and cocaine, with delusions, especially persecutory, and auditory hallucinations being the main reported symptoms. Although the pathophysiology of these disorders is not completely understood, there is evidence that the use of illicit drugs is a risk factor for the development of psychotic events, therefore, the knowledge of the factors involved in this relationship is important in clinical practice. In conclusion, it is important that health professionals know that the use of recreational drugs can lead to the development of psychosis and that they know how to assess the best way to approach these patients so that they can offer the best possible support.
\end{abstract}

Keywords: Psychosis; Psychotic disorders; Substances; Recreational drugs.

\section{Resumen}

Psicosis es un término amplio que caracteriza una serie de trastornos que tienen en común la producción de los llamados síntomas psicóticos, principalmente delirios y alucinaciones. Los trastornos psicóticos se pueden dividir en primarios y secundarios, incluyendo en esta última categoría los trastornos psicóticos inducidos por sustancias, entre los que destacan las drogas recreativas. El propósito de esta revisión fue analizar las principales características fisiopatológicas, sintomáticas, diagnósticas y terapéuticas de los trastornos psicóticos relacionados con el uso de 
drogas recreativas. Se realizó una búsqueda de trabajos previos en las plataformas SciELO, Lilacs y PubMed y se incluyeron un total de 27 artículos científicos luego de aplicar criterios de inclusión y exclusión. A través de los estudios analizados se observó que las drogas más frecuentemente asociadas a los trastornos psicóticos fueron el cannabis, las anfetaminas, el alcohol y la cocaína, siendo los delirios, especialmente persecutorios, y las alucinaciones auditivas los principales síntomas reportados. Si bien la fisiopatología de estos trastornos no se comprende completamente, existe evidencia de que el uso de drogas ilícitas es un factor de riesgo para el desarrollo de eventos psicóticos, por lo que el conocimiento de los factores involucrados en esta relación es importante en la práctica clínica. En conclusión, es importante que los profesionales de la salud sepan que el uso de drogas recreativas puede conducir al desarrollo de psicosis y que sepan evaluar la mejor forma de acercarse a estos pacientes para que puedan ofrecer el mejor apoyo posible.

Palabras clave: Psicosis; Trastornos psicóticos; Sustancias; Drogas recreativas.

\section{Introdução}

A psicose é uma síndrome clínica associada a deficiências em vários domínios que incluem pensamento, comportamento e cognição (Kilciksiz, Keefe, Benoit, Öngür, \& Torous, 2020). Psicose é um termo amplo que engloba um espectro que vai desde o Transtorno Psicótico Breve até Esquizofrenia. De um modo geral, um transtorno que seja acompanhado de delírios, alucinações, pensamento desorganizado, comportamento motor grosseiramente desorganizado ou anormal e/ou sintomas negativos (expressão emocional diminuída e avolia, por exemplo), pode ser considerado como psicótico (American Psychiatric Association [APA], 2013).

Os transtornos psicóticos primários compreendem doenças em que os sintomas psicóticos não surgem em decorrência de uma condição clínica base, como Transtornos de Humor ou Transtorno Esquizoafetivo. Dentre os Transtornos Psicóticos Primários, destaca-se a Esquizofrenia (Tampi, Young, Hoq, Resnik, \& Tampi, 2019). As desordens psicóticas secundárias incluem a psicose como sintoma de outros distúrbios, incluindo desordens neurocognitivas, delírio, uso de substâncias ilícitas, ou outros medicamentos e desordens neurológicas (Tampi et al., 2019).

A psicose é um transtorno relativamente frequente, com uma incidência estimada entre 1.5 e 6.5 por 100000 pessoas por ano, comparável com a incidência de doenças psiquiátricas muito conhecidas, tais como Transtorno Bipolar de Humor e Transtorno Esquizoafetivo (Murrie, Lappin, Large, \& Sara, 2020) e aproximadamente 25\% de todos os pacientes que apresentam o primeiro episódio psicótico com necessidade de atendimento de emergência recebem o diagnóstico de Transtorno Psicótico Induzido por Substância (TPIS) (APA, 2013; Murrie et al., 2020).

O TPIS é caracterizado pelo surgimento de uma síndrome psicótica com sintomas positivos (delírios e/ou alucinações) que se desenvolvem durante ou após o uso de uma substância capaz de produzi-los, excluindo-se doenças psiquiátricas primárias que poderiam causar alterações clínicas semelhantes (APA, 2013). Os sintomas psicóticos se resolvem espontaneamente em algumas horas, dias ou semanas (APA, 2013; Murrie et al., 2020), apesar de haver uma quantidade significativa de relatos em que as manifestações podem durar meses ou anos (Murrie et al., 2020; Palma-Álvarez, RosCucurull, Ramos-Quiroga, Roncero, \& Grau-López, 2019; Redes, Brescia, Wschebor, \& Gutiérrez, 2013; Kurtom, Henning, \& Espiridion, 2019).

Em 2007, cerca de 200 milhões de indivíduos entre os 15 e os 64 anos de idade eram usuários de drogas recreativas no mundo (United Nations Office of Drug and Crime [UNODC], 2019). Estima-se que 275 milhões de indivíduos em todo o mundo, ou 5,6\% da população global com idade entre 16 a 54 anos de idade, usaram uma droga ilícita pelo menos uma vez em 2016 (Ryan, Smeltzer, \& Sharts-Hopko, 2019). Estima-se que 450.000 pessoas morreram em consequência do uso de drogas; 167.750 diretamente de transtornos por uso de drogas, e o restante de doenças relacionadas ao uso de drogas, como o vírus da hepatite C e o vírus da imunodeficiência humana (Ryan et al., 2019). No Brasil, em 2005, estimou-se que 22,8\% dos brasileiros já haviam usado drogas recreativas, o que correspondeu a cerca de 46 mil indivíduos (Carlini et al., 2006). Em 2015, entre indivíduos com idade entre 12 a 65 anos de idade, 153.095 mil eram usuários de drogas (Fundação Oswaldo Cruz 
[FIOCRUZ], \& Instituto de Comunicação e Informação Científica e Tecnológica em Saúde [ICICT], 2017). No Brasil e ao redor do mundo, a droga recreativa mais utilizada é a cannabis (UNODC, 2019; FIOCRUZ \& ICICT, 2017), com cerca de 192 milhões de usuários em todo o mundo, enquanto os opioides continuam a causar os maiores danos, sendo responsáveis por $76 \%$ das mortes relacionadas ao uso de drogas (Ryan et al., 2019).

Existem evidências de que o uso de drogas recreativas, especialmente a cannabis sejam um fator de risco para o surgimento de Transtornos Psicóticos Crônicos, como a esquizofrênia (Björkenstam, Björkenstam, Hjern, Reutfors, \& Bodén, 2013; Marconi, Di Forti, Lewis, Murray, \& Vassos, 2016). As drogas recreativas mais frequentemente associadas com psicose além da cannabis são: álcool, alucinógenos, cocaína e anfetaminas (Rounsaville, 2007; Thirthalli \& Benegal, 2006; APA, 2013). Considerando que a frequência do uso de drogas, especialmente em adolescentes e jovens adultos, torna-se cada vez maior (UNODC, 2019; FIOCRUZ \& ICICT, 2017) é possível que os profissionais de saúde se deparem cada vez mais com episódios psicóticos induzidos por essas substâncias nas emergências e clínicas de saúde mental (Fielitz et al., 2010).

Não existe um consenso acerca da fisiopatologia do TPIS, sabe-se, entretanto, que algumas das drogas recreativas, tais como álcool, cannabis, alucinógenos, cocaína e anfetaminas produzem certos padrões de ativação e inibição de vias neuronais e neurotransmissão semelhantes aos encontrados na esquizofrenia (Ham, Kim, Chung, \& Im, 2017; Müller, Dolder, Schmidt, Liechti, \& Borgwardt, 2018; Winton Brow et al., 2011). Desta forma, o objetivo dessa revisão de literatura foi analisar as principais características fisiopatológicas, sintomáticas, diagnósticas e terapêuticas dos transtornos psicóticos relacionados ao uso de drogas recreativas.

\section{Metodologia}

Trata-se de um estudo de abordagem qualitativa, retrospectiva e transversal executado por meio de uma revisão integrativa da literatura. As bases de dados utilizadas foram o SciELO, LILACS e a National Library of Medicine (PubMed). A busca pelos artigos foi realizada considerando os descritores "substance", "induced" e "psychosis", utilizando o operador booleano "AND". A revisão de literatura foi realizada seguindo as seguintes etapas: estabelecimento do tema; definição dos parâmetros de elegibilidade; definição dos critérios de inclusão e exclusão; verificação das publicações nas bases de dados; exame das informaç̃os encontradas; análise dos estudos encontrados e exposição dos resultados (Pereira, Shitsuka, Parreira, \& Shitsuka, 2018; Silva et al., 2018). Foram incluídos no estudo artigos publicados nos últimos 10 anos (2010-2020); nos idiomas inglês, português, sueco, húngaro e espanhol; de acesso livre e artigos cujos estudos eram do tipo ensaio clínico, estudo clínico randomizado controlado ou relato de caso. Foram excluídos os artigos que não tinham definição clara de embasamento teórico e temático afinado aos objetos do estudo, que não trabalhavam a relação das substâncias com as psicoses propriamente ditas e artigos fora do tema abordado.

\section{Resultados}

A busca resultou em um total de 970 trabalhos. Foram encontrados 894 artigos na base de dados PubMed, 69 artigos no LILACS e 7 artigos na base de dados SciELO. Após a aplicação dos critérios de inclusão e exclusão foram selecionados 16 artigos na base de dados PubMed, 7 artigos no LILACS e 4 artigos no SciELO, sendo que 1 artigo foi retirado por estar duplicado entre as plataformas LILACS e SciELO, conforme apresentado na Figura 1. 
Figura 1. Fluxograma de identificação e seleção dos artigos selecionados nas bases de dados PubMed, SciELO e LILACS.

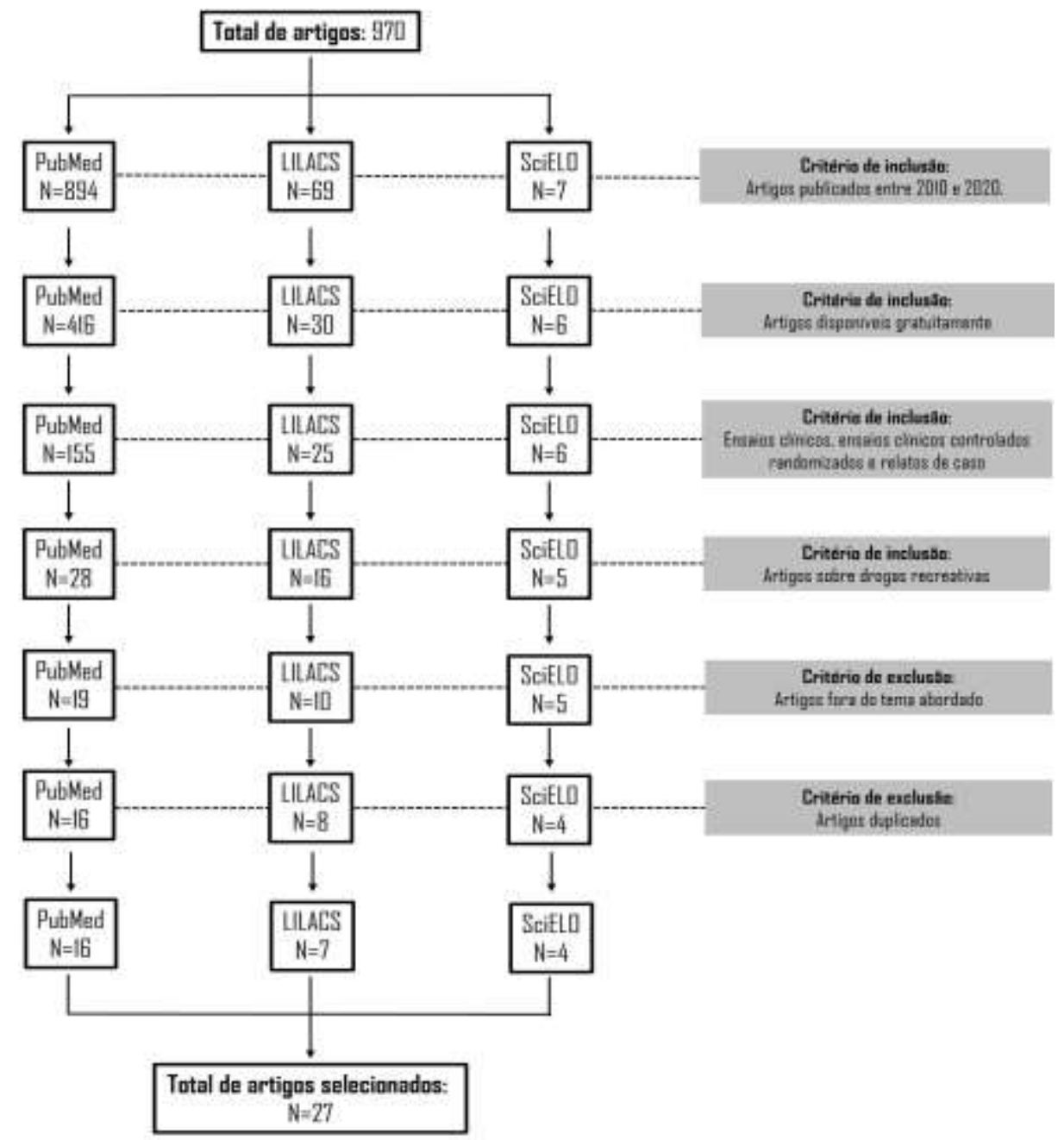

Fonte: Autores (2021).

Dos 27 estudos selecionados 14 são relatos de caso, 4 são séries de casos, 7 são ensaios clínicos e 2 são estudos clínicos randomizados controlados (Tabela 1). Dos artigos selecionados, vinte e cinco estudos observaram que os pacientes com sintomas psicóticos fizeram uso de drogas recreativas tais como: cannabis, álcool, metanfetamina, cocaína, mefedrona, ayahuasca, óxido nítrico, heroína, anfetaminas e crack. Destes estudos as principais drogas causadoras de sintomas psicóticos foram a cannabis, anfetaminas, o álcool e a cocaína. Outras drogas como ayahuasca, mefedrona, óxido nítrico, heroína e crack foram citadas em apenas um estudo cada uma conforme apresentado na Figura 2. Quatro estudos não observaram sintomas psicóticos em indivíduos após o uso de óxido nítrico, metanfetamina, cocaína e cannabis. 
Tabela 1. Caracterização dos artigos conforme ano de publicação, tipo de estudo e principais conclusões.

\begin{tabular}{|c|c|c|c|c|}
\hline Autor & Ano & Título & Tipo de estudo & Principais conclusões \\
\hline $\begin{array}{l}\text { B. Zhao, L. } \\
\text { Zhao, Li, \& } \\
\text { R. Zhao }\end{array}$ & 2020 & $\begin{array}{l}\text { Subacute Combined } \\
\text { Degeneration Induced by } \\
\text { Nitrous Oxide Inhalation: } \\
\text { Two Case Reports }\end{array}$ & $\begin{array}{l}\text { Série de casos } \\
\qquad(\mathrm{n}=2)\end{array}$ & $\begin{array}{l}\text { Os dois pacientes apresentaram } \\
\text { neurológicos, mas não apresentaram } \\
\text { psicóticos ou psiquiátricos de um modo geral. }\end{array}$ \\
\hline Kurtom et al. & 2019 & $\begin{array}{l}\text { Hallucinogen-persisting } \\
\text { Perception Disorder in a 21- } \\
\text { year-old Man }\end{array}$ & $\begin{array}{l}\text { Relato de caso } \\
\qquad(\mathrm{n}=1)\end{array}$ & $\begin{array}{l}\text { Alucinógenos podem causar episódios psicóticos } \\
\text { prolongados para além de intoxicação aguda. }\end{array}$ \\
\hline $\begin{array}{l}\text { Albobali \& } \\
\text { Madi }\end{array}$ & 2019 & $\begin{array}{l}\text { Polysubstance Use Disorder } \\
\text { After Sleeve Gastrectomy }\end{array}$ & $\begin{array}{l}\text { Relato de caso } \\
\qquad(\mathrm{n}=1)\end{array}$ & $\begin{array}{l}\text { Um quadro psicótico grave foi associado ao uso de } \\
\text { cannabis, álcool e metanfetamina. }\end{array}$ \\
\hline Ikawa et al. & 2019 & $\begin{array}{l}\text { A case of methamphetamine } \\
\text { use disorder presenting a } \\
\text { condition of ultra-rapid cycler } \\
\text { bipolar disorder }\end{array}$ & $\begin{array}{l}\text { Relato de caso } \\
\qquad(\mathrm{n}=1)\end{array}$ & $\begin{array}{l}\text { O uso de metanfetamina não causou sintomas } \\
\text { psicóticos, mas se associou à uma forma de } \\
\text { transtorno bipolar de humor mais agressiva. }\end{array}$ \\
\hline $\begin{array}{l}\text { Gerlach et } \\
\text { al. }\end{array}$ & 2019 & $\begin{array}{l}\text { Clinical challanges in patients } \\
\text { whith first episode psychosis } \\
\text { and cannabis use: mini- } \\
\text { review and case study }\end{array}$ & $\begin{array}{l}\text { Série de casos } \\
\qquad(\mathrm{n}=5)\end{array}$ & $\begin{array}{l}\text { Todos os pacientes apresentaram sintomas psicóticos } \\
\text { graves a ponto de requerer internação, todos testaram } \\
\text { positivo para THC no momento da internação e } \\
\text { relataram uso prévio de cannabis por pelo menos } 6 \\
\text { anos. }\end{array}$ \\
\hline $\begin{array}{l}\text { Palma- } \\
\text { Àlvarez et } \\
\text { al. }\end{array}$ & 2019 & $\begin{array}{l}\text { Cocaine-induced psychosis } \\
\text { and ansenapine as treatment: } \\
\text { a case Study }\end{array}$ & $\begin{array}{l}\text { Relato de caso } \\
\qquad(\mathrm{n}=1)\end{array}$ & $\begin{array}{l}\text { Um quadro psicótico refratário e prolongado foi } \\
\text { associado ao uso de cocaína e só foi controlado com } \\
\text { ansenapina, um antipsicótico atípico. }\end{array}$ \\
\hline $\begin{array}{l}\text { Zamora- } \\
\text { Rodríguez, } \\
\text { Sánchez- } \\
\text { Walsen- } \\
\text { Hernández, } \\
\text { Guisado- } \\
\text { Macias, \& } \\
\text { Vaz-Leal }\end{array}$ & 2018 & $\begin{array}{l}\text { El uso de sustancias y su } \\
\text { relación con el curso del } \\
\text { trastorno bipolar }\end{array}$ & $\begin{array}{l}\text { Ensaio clínico } \\
\quad(\mathrm{n}=394)\end{array}$ & $\begin{array}{l}\text { Os pacientes bipolares que usavam drogas recreativas } \\
\text { tiveram maior frequência de episódios psicóticos em } \\
\text { relação aos bipolares não usuários. }\end{array}$ \\
\hline Müller et al. & 2018 & $\begin{array}{l}\text { Altered network hub } \\
\text { connectivity after acute LSD } \\
\text { administration }\end{array}$ & $\begin{array}{l}\text { Estudo clínico } \\
\text { randomizado } \\
\text { controlado } \\
\quad(\mathrm{n}=20)\end{array}$ & $\begin{array}{l}\text { A administração de LSD induziu uma atividade } \\
\text { neuronal semelhante a estados de psicose, o que não } \\
\text { se repetiu no grupo controle. }\end{array}$ \\
\hline Haq et al. & 2017 & $\begin{array}{l}\text { "Spice" (Synthetic Marijuana) } \\
\text { Induced Acute Myocardial } \\
\text { Infarction: A Case Series }\end{array}$ & $\begin{array}{l}\text { Série de casos } \\
\qquad(\mathrm{n}=3)\end{array}$ & $\begin{array}{l}\text { Em nenhum dos } 3 \text { pacientes houve relatos de } \\
\text { sintomas psicóticos ou psiquiátricos de um modo } \\
\text { geral. }\end{array}$ \\
\hline $\begin{array}{l}\text { Neyra- } \\
\text { Ontaneda }\end{array}$ & 2017 & $\begin{array}{l}\text { Psicosis inducida por } \\
\text { ayahuasca: reporte de un caso. }\end{array}$ & $\begin{array}{l}\text { Relato de caso } \\
\quad(\mathrm{n}=1)\end{array}$ & $\begin{array}{l}\text { Um quadro prolongado e grave de psicose foi } \\
\text { causado pelo uso de um alucinógeno ritual, o chá de } \\
\text { ayahuasca. }\end{array}$ \\
\hline $\begin{array}{l}\text { Thomas, } \\
\text { Lategan, } \\
\text { Verster, } \\
\text { Kidd, \& } \\
\text { Weich }\end{array}$ & 2016 & $\begin{array}{l}\text { Methamphetamine-induced } \\
\text { psychosis: Clinical features, } \\
\text { treatment modalities and } \\
\text { outcomes }\end{array}$ & $\begin{array}{l}\text { Ensaio Clínico } \\
\qquad(\mathrm{n}=56)\end{array}$ & $\begin{array}{l}\text { Todos os pacientes incluídos no estudo apresentaram } \\
\text { episódios psicóticos induzidos por metanfetamina, } \\
\text { entretanto mais da metade dos pacientes referiram } \\
\text { também uso de outras substâncias nos últimos } 3 \\
\text { meses, especialmente cannabis. }\end{array}$ \\
\hline $\begin{array}{l}\text { Tucker, } \\
\text { Kekulawala, } \\
\text { Kent, } \\
\text { Mostafa, \& } \\
\text { Harvey }\end{array}$ & 2016 & $\begin{array}{l}\text { Polysubstance-induced } \\
\text { relapse of schizoaffective } \\
\text { disorder refractory to high- } \\
\text { doserantipsychotic } \\
\text { medications: a case report }\end{array}$ & $\begin{array}{l}\text { Relato de caso } \\
\qquad(\mathrm{n}=1)\end{array}$ & $\begin{array}{l}\text { Houve uma reativação rica em sintomas psicóticos do } \\
\text { transtorno esquizoafetivo do paciente após uso de } \\
\text { metanfetamina e cannabis. }\end{array}$ \\
\hline
\end{tabular}




\begin{tabular}{|c|c|c|c|c|}
\hline $\begin{array}{l}\text { Mancke, } \\
\text { Kaklauskaitè } \\
\text {, Kollmer, \& } \\
\text { Weiler }\end{array}$ & 2016 & $\begin{array}{l}\text { Psychiatric comorbidities in a } \\
\text { young man with subacute } \\
\text { myelopathy induced by } \\
\text { abusive nitrous oxide } \\
\text { consumption: a case report }\end{array}$ & $\begin{array}{l}\text { Relato de caso } \\
\qquad(\mathrm{n}=1)\end{array}$ & $\begin{array}{l}\text { O paciente sofreu uma síndrome neurológica } \\
\text { associada a inalação de óxido nítrico, mas houve } \\
\text { relato de episódio psicótico prévio induzido por } \\
\text { formulação de cannabis em alta concentração. }\end{array}$ \\
\hline $\begin{array}{l}\text { Mancheno et } \\
\text { al. }\end{array}$ & 2016 & $\begin{array}{l}\text { Prevalencia de patología dual } \\
\text { en el centro de reposo y } \\
\text { adicciones (CRA). Cuenca, } \\
\text { abril - deciembre } 2016 \text {. }\end{array}$ & $\begin{array}{l}\text { Ensaio Clínico } \\
\qquad(\mathrm{n}=133)\end{array}$ & $\begin{array}{l}21,1 \% \text { dos pacientes com transtornos por uso de } \\
\text { substâncias possuíam também transtornos psicóticos } \\
\text { na amostra avaliada. }\end{array}$ \\
\hline $\begin{array}{l}\text { Revilla- } \\
\text { Zúñiga, } \\
\text { Rivera- } \\
\text { Encinas, \& } \\
\text { Cruzado }\end{array}$ & 2016 & $\begin{array}{l}\text { Diagnóstico erróneo de } \\
\text { psicosis inducida por cocaína } \\
\text { en una persona con } \\
\text { esquizofrenia y masticadora } \\
\text { de hojas de coca }\end{array}$ & $\begin{array}{l}\text { Relato de caso } \\
\qquad(\mathrm{n}=1)\end{array}$ & $\begin{array}{l}\text { O paciente apresentava critérios para um quadro } \\
\text { psicótico primário de esquizofrenia embora tenham } \\
\text { sido encontradas evidências de cocaína no organismo } \\
\text { do paciente. }\end{array}$ \\
\hline $\begin{array}{l}\text { Barrio, } \\
\text { Gaskell, } \\
\text { Goti, } \\
\text { Vilardell, \& } \\
\text { Fàbregas }\end{array}$ & 2016 & $\begin{array}{l}\text { Síntomas psicóticos } \\
\text { persistentes después del } \\
\text { uso abusivo prolongado de } \\
\text { mefedrona: una serie de } \\
\text { dos casos }\end{array}$ & $\begin{array}{l}\text { Serie de casos } \\
\qquad(\mathrm{n}=2)\end{array}$ & $\begin{array}{l}\text { O uso crônico de mefedrona, uma droga semelhante } \\
\text { às anfetaminas, pode causar sintomas psicóticos a } \\
\text { longo prazo. }\end{array}$ \\
\hline Chin et al. & 2015 & $\begin{array}{l}\text { Rehabilitation essential in the } \\
\text { recovery of multifactorial } \\
\text { subacute } \\
\text { degeneration }\end{array}$ & $\begin{array}{l}\text { Relato de caso } \\
\qquad(\mathrm{n}=1)\end{array}$ & $\begin{array}{l}\text { Sintomas psicóticos e neurológicos graves foram } \\
\text { causados pelo uso de álcool e inalação de óxido } \\
\text { nítrico. }\end{array}$ \\
\hline $\begin{array}{l}\text { Dannatt, } \\
\text { Cloete, } \\
\text { Kidd, \& } \\
\text { Weich }\end{array}$ & 2014 & $\begin{array}{l}\text { Frequency and correlates of } \\
\text { comorbid psychiatric illness } \\
\text { in patients with heroin use } \\
\text { disorder admitted to Stikland } \\
\text { Opioid Detoxification Unit, } \\
\text { South Africa }\end{array}$ & $\begin{array}{l}\text { Ensaio Clínico } \\
\quad(\mathrm{n}=141)\end{array}$ & $\begin{array}{l}\text { Episódios prévios de psicose induzida por substância } \\
\text { foi a comorbidade mais frequentemente observada } \\
\text { nos pacientes incluídos no estudo, todos dependentes } \\
\text { de heroína. }\end{array}$ \\
\hline $\begin{array}{l}\text { Guimarães et } \\
\text { al. }\end{array}$ & 2014 & $\begin{array}{l}\text { Suicide risk and alcohol and } \\
\text { drug abuse in outpatients with } \\
\text { HIV infection and Chagas } \\
\text { disease }\end{array}$ & $\begin{array}{l}\text { Ensaio clínico } \\
\qquad(\mathrm{n}=125)\end{array}$ & $\begin{array}{l}\text { Mais de } 30 \% \text { dos pacientes era dependente de álcool } \\
\text { ou outras substâncias, sendo que } 19,2 \% \text { já haviam } \\
\text { apresentado algum tipo de evento psicótico. }\end{array}$ \\
\hline $\begin{array}{l}\text { Personne, } \\
\text { Westerberg, } \\
\text { \& Hammer- } \\
\text { Pettersen }\end{array}$ & 2014 & $\begin{array}{l}\gg \text { Spice« } \quad-\quad \text { syntetiska } \\
\text { kannabinoider med riskabla } \\
\text { effekter }\end{array}$ & $\begin{array}{l}\text { Relato de caso } \\
\quad(\mathrm{n}=1)\end{array}$ & $\begin{array}{l}\text { O paciente não apresentou sintomas psicóticos } \\
\text { durante a intoxicação por uma formulação de } \\
\text { cannabis em alta concentração. }\end{array}$ \\
\hline $\begin{array}{l}\text { Filho \& } \\
\text { Silveira }\end{array}$ & 2014 & $\begin{array}{l}\text { Psicose e drogadição: a } \\
\text { construção do caso na clínica } \\
\text { de enfermagem }\end{array}$ & $\begin{array}{l}\text { Relato de caso } \\
\qquad(\mathrm{n}=1)\end{array}$ & $\begin{array}{l}\text { Um quadro psicótico se estabeleceu com uso } \\
\text { prolongado de álcool. }\end{array}$ \\
\hline $\begin{array}{l}\text { Chan, Wood, } \\
\text { Hudson, \& } \\
\text { Dargan }\end{array}$ & 2013 & $\begin{array}{l}\text { Acute psychosis associated } \\
\text { with recreational use of } \\
\text { benzofuran } 6-(2- \\
\text { aminopropyl)benzofuran }(6- \\
\text { APB) and cannabis }\end{array}$ & $\begin{array}{l}\text { Relato de caso } \\
\qquad(\mathrm{n}=1)\end{array}$ & $\begin{array}{l}\text { Um quadro psicótico grave e prolongado foi } \\
\text { associado ao uso de cannabis e 6-APB, uma } \\
\text { anfetamina. }\end{array}$ \\
\hline Redes et al. & 2013 & $\begin{array}{l}\text { Acerca de un caso clínico de } \\
\text { patología dual, de psicosis y } \\
\text { consumo de sustancias. }\end{array}$ & $\begin{array}{l}\text { Relato de caso } \\
\qquad(\mathrm{n}=1)\end{array}$ & $\begin{array}{l}\text { Os episódios psicóticos foram associados a } \\
\text { alucinógenos, cannabis e/ou cocaína, não } \\
\text { necessariamente todas em conjunto nas mesmas } \\
\text { internações. }\end{array}$ \\
\hline $\begin{array}{l}\text { Fullajtar \& } \\
\text { Ferencz }\end{array}$ & 2012 & $\begin{array}{l}\text { Dizájner drog indukálta } \\
\text { pszichózis }\end{array}$ & $\begin{array}{l}\text { Relato de caso } \\
\qquad(\mathrm{n}=1)\end{array}$ & $\begin{array}{l}\text { O uso regular de 3,4-metilenodioxipirovalerona, uma } \\
\text { anfetamina, causou um episódio psicótico prolongado } \\
\text { para além da intoxicação aguda pela substância. }\end{array}$ \\
\hline
\end{tabular}


Research, Society and Development, v. 10, n. 2, e21910212459, 2021

(CC BY 4.0) | ISSN 2525-3409 | DOI: http://dx.doi.org/10.33448/rsd-v10i2.12459

\begin{tabular}{|c|c|c|c|c|}
\hline $\begin{array}{l}\text { Winton } \\
\text { Brown et al. }\end{array}$ & 2011 & $\begin{array}{l}\text { Modulation of auditory and } \\
\text { visual processing by delta-9- } \\
\text { tetrahydrocannabinol and } \\
\text { cannabidiol: an fMRI study }\end{array}$ & $\begin{array}{l}\text { Estudo Clínico } \\
\text { Randomizado } \\
\text { Controlado } \\
\quad(\mathrm{n}=14)\end{array}$ & $\begin{array}{l}\text { A administração de THC induziu um padrão de } \\
\text { ativação neuronal e sintomas correspondentes com } \\
\text { estados de psicose, o que não se repetiu no grupo } \\
\text { controle. }\end{array}$ \\
\hline Fielitz et al. & 2010 & \begin{tabular}{lrr} 
Consumo de & sustancias \\
psicoactivas en pacientes con \\
trastornos & psicóticos \\
ingresados en & Sala de \\
Emergencia & psiquiátrica. \\
Hospital & \multicolumn{2}{c}{ Vilardebó. } \\
Montevideo. Uruguay
\end{tabular} & $\begin{array}{l}\text { Ensaio clínico } \\
\qquad(\mathrm{n}=181)\end{array}$ & $\begin{array}{l}17,9 \% \text { dos pacientes que foram atendidos no serviço } \\
\text { de emergência psiquiátrica por sintomas psicóticos } \\
\text { tinham indícios na urina de uso recente de cannabis } \\
\text { e/ou cocaína. }\end{array}$ \\
\hline Fontanella & 2010 & $\begin{array}{l}\text { Sintomas psicóticos e } \\
\text { cognitivos associados à busca } \\
\text { de tratamento por } \\
\text { dependentes de substâncias - } \\
\text { Um estudo qualitativo }\end{array}$ & $\begin{array}{l}\text { Ensaio clínico } \\
\qquad(\mathrm{n}=13)\end{array}$ & $\begin{array}{l}\text { Cinco dos pacientes com sintomas psicóticos eram } \\
\text { dependentes de cocaína, seis deles eram dependentes } \\
\text { de álcool, um era dependente de cannabis e um era } \\
\text { dependente de crack. }\end{array}$ \\
\hline
\end{tabular}

* THC: tetrahidrocanabinol; LSD: dietilamida do ácido lisérgico. Fonte: Autores (2021).

Figura 2. Número de estudos que associaram o uso das drogas recreativas ao desenvolvimento de sintomas psicóticos.

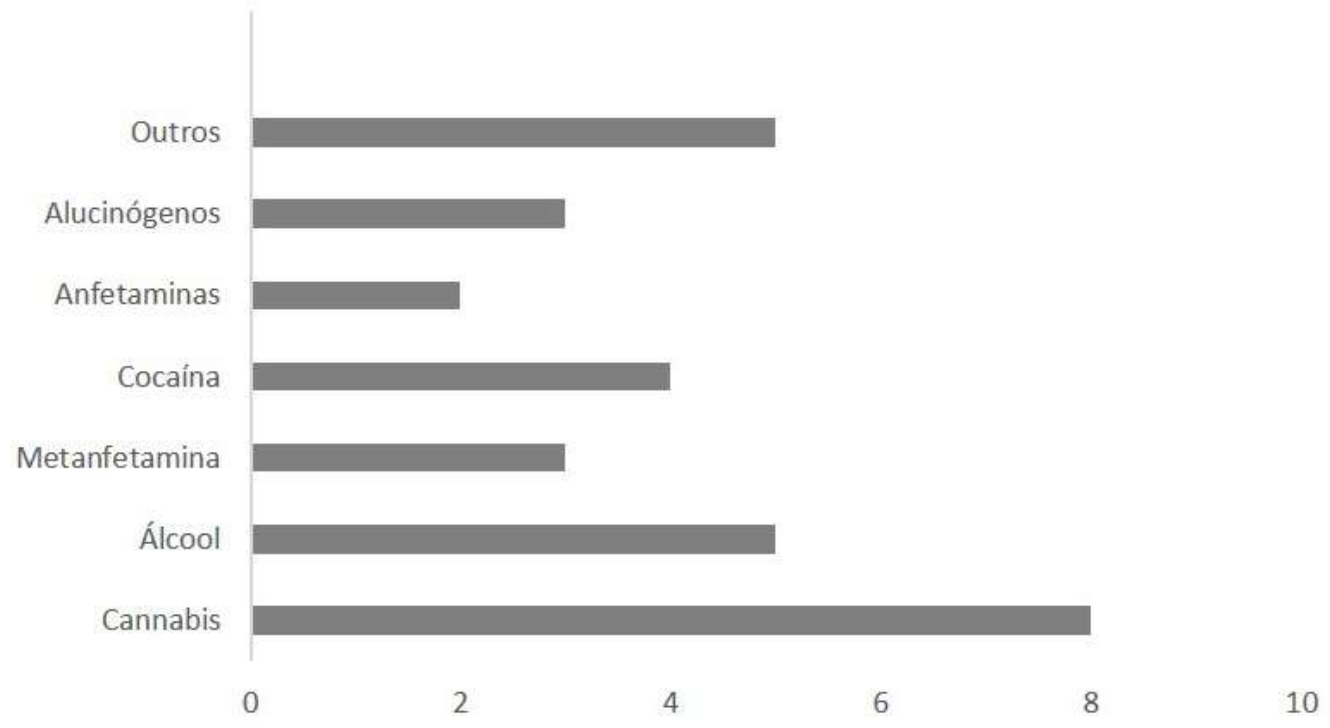

Número de estudos

Fonte: Autores (2021).

Quanto aos sintomas em 18 dos 27 trabalhos houve relato específico de sintomas positivos nos pacientes expostos à cannabis, cocaína, álcool, alucinógenos ou substâncias anfetamínicas (Tabela 2). Destes, 13 descreveram delírios de conteúdo paranoide ou persecutório, 4 de conteúdo grandioso, incluindo delírios místicos e de ressuscitação e 3 descreveram delírios de referência. Dez trabalhos relataram vivências alucinatórias tendo como principal droga causadora a cocaína, que foi citada em quatro trabalhos, seguida de cannabis, alucinógenos e anfetaminas, associadas em três trabalhos cada uma. As alucinações foram especificadas como auditivas em sete artigos e como visuais em três. As alucinações cinestésicas e cenestésicas foram relatadas 1 vez e 2 vezes, respectivamente. Sintomas negativos foram relatados em apenas 3 artigos. Agressividade e/ou 
agitação psicomotora estavam presentes em 11 trabalhos, tendo sido mais frequentemente relatadas em relação à psicose causada por cannabis, anfetaminas e cocaína, respectivamente.

Tabela 2. Principais sintomas apresentados pelos pacientes expostos a drogas recreativas nos artigos avaliados.

\begin{tabular}{|c|c|c|c|}
\hline Autor & Ano & Substância utilizada & Sintomas apresentados pelos pacientes \\
\hline Zhao et al. & 2020 & Óxido nítrico & $\begin{array}{l}\text { Paresia e parestesia progressivas nos membros e ataxia. Não houve sintomas } \\
\text { psiquiátricos no momento do relato. }\end{array}$ \\
\hline Kurtom et al. & 2019 & LSD & $\begin{array}{l}\text { Flashbacks, alucinações cenestésicas, déficits cognitivos e embotamento } \\
\text { social. }\end{array}$ \\
\hline Albobali \& Madi & 2019 & $\begin{array}{l}\text { Fenetilina, cannabis, álcool } \\
\text { e metanfetamina }\end{array}$ & $\begin{array}{l}\text { Ideação paranóica, alucinações auditivas, insônia grave e comportamento } \\
\text { agressivo. }\end{array}$ \\
\hline Ikawa et al. & 2019 & Metanfetamina & $\begin{array}{l}\text { Ansiedade, humor depressivo e reconhecimento pessimista, irritabilidade } \\
\text { extrema, apatia, fala pressionada, pensamentos acelerados e comportamento } \\
\text { estereotipado. }\end{array}$ \\
\hline Gerlach et al. & 2019 & Tetrahidrocanabidiol & $\begin{array}{l}\text { Agitação extrema, confusão, desorganização, comportamento perturbador, } \\
\text { imprevisível, irritável, preocupações paranoicas, agressividade, humor } \\
\text { elevado, inquietação. }\end{array}$ \\
\hline $\begin{array}{l}\text { Palma-Àlvarez et } \\
\text { al.r }\end{array}$ & 2019 & Cocaína & $\begin{array}{l}\text { Alucinações auditivas e cinestésicas, delírios persecutórios, irritabilidade, } \\
\text { ansiedade e inquietação psicomotora. }\end{array}$ \\
\hline $\begin{array}{l}\text { Zamora-Rodríguez } \\
\text { et al. }\end{array}$ & 2018 & $\begin{array}{l}\text { Álcool, cocaína, opioides, } \\
\text { cannabis e sedativos }\end{array}$ & ------------ \\
\hline Müller et al. & 2018 & LSD & ------------ \\
\hline Haq et al. & 2017 & Cannabis sintética & $\begin{array}{l}\text { Dor torácica lancinante com irradiação para membro superior esquerdo e dor } \\
\text { torácica retroesternal sem irradiação. Não houve sintomas psiquiátricos no } \\
\text { momento do relato. }\end{array}$ \\
\hline Neyra-Ontaneda & 2017 & Ayahuasca & $\begin{array}{l}\text { Comportamento violento, delírios paranoides e de ressuscitação, discurso } \\
\text { desorganizado e alucinações visuais, táteis e cenestésicas. }\end{array}$ \\
\hline Thomas et al. & 2016 & Metanfetamina & $\begin{array}{l}\text { Alucinações, comportamento agressivo, delírios paranoides e de } \\
\text { grandiosidade. Embotamento afetivo e outros sintomas negativos foram } \\
\text { encontrados em poucos pacientes. }\end{array}$ \\
\hline Tucker et al. & 2016 & Cannabis e metanfetamina & Comportamento desorganizado, agressivo e suicida e delírios paranoides. \\
\hline Mancke et al. & 2016 & Óxido Nítrico & $\begin{array}{l}\text { Parestesias em membros e mãos, dificuldade na marcha e déficit de } \\
\text { equilíbrio. Não houve sintomas psiquiátricos no momento do relato. }\end{array}$ \\
\hline Mancheno et al. & 2016 & $\begin{array}{l}\text { Substâncias não } \\
\text { especificadas }\end{array}$ & ------------- \\
\hline $\begin{array}{l}\text { Revilla-Zúñiga et } \\
\text { al. }\end{array}$ & 2016 & Cocaína & $\begin{array}{l}\text { Comportamento agressivo, delírios paranoides e de grandiosidade e } \\
\text { alucinações auditivas. }\end{array}$ \\
\hline Barrio et al. & 2016 & Mefedrona & Delírios paranoides e de referência e alucinações auditivas. \\
\hline Chin et al. & 2015 & Óxido nítrico & Delírios paranoides e alucinações visuais e auditivas. \\
\hline Dannat et al. & 2014 & Heroína & ------------- \\
\hline Guimarães et al. & 2014 & Álcool & ------------ \\
\hline Personne et al. & 2014 & Cannabis sintética & $\begin{array}{l}\text { Atividade convulsiva. Não houve sintomas psiquiátricos no momento do } \\
\text { relato. }\end{array}$ \\
\hline Filho \& Silveira & 2014 & Álcool & Comportamento desorganizado e delírios de referência. \\
\hline
\end{tabular}


Research, Society and Development, v. 10, n. 2, e21910212459, 2021

(CC BY 4.0) | ISSN 2525-3409 | DOI: http://dx.doi.org/10.33448/rsd-v10i2.12459

\begin{tabular}{l|l|c|l}
\hline Chan et al. & 2013 & Cannabis e anfetamina & Comportamento agitado, automutilação, delírios paranoides. \\
\hline Redes et al. & 2013 & $\begin{array}{c}\text { Alucinógenos, cannabis e } \\
\text { cocaína }\end{array}$ & $\begin{array}{l}\text { Comportamento agitado e agressivo, delírios paranoides e místicos, discurso } \\
\text { desorganizado e alucinações auditivas. }\end{array}$ \\
\hline Fullajtar \& Ferencz & 2012 & Anfetamina & Delírios paranoides e de referência. \\
\hline Winton et al. & 2011 & $\begin{array}{c}\text { delta-9- } \\
\text { tetrahydrocannabinol e } \\
\text { cannabidiol }\end{array}$ & Ansiedade e sintomas psicóticos positivos não especificados. \\
\hline Fielitz et al. & 2010 & $\begin{array}{c}\text { Cannabis, pasta base de } \\
\text { cocaína e cocaína. }\end{array}$ & Sintomas psicóticos não especificados e comportamento agressivo. \\
\hline Fontanella & 2010 & $\begin{array}{c}\text { Cocaína, álcool, cannabis e } \\
\text { crack. }\end{array}$ & Delírios paranoides, déficits cognitivos e alucinações visuais. \\
\hline
\end{tabular}

Fonte: Autores (2021).

\section{Discussão}

Os resultados deste estudo mostraram que dos vinte e sete artigos selecionados apenas quatro não observaram associação entre o uso de drogas recreativas e o desenvolvimento de psicose, sugerindo que o uso destas substâncias ilícitas está relacionado aos sintomas psicóticos. Não há consenso na literatura médica sobre a fisiopatologia exata da esquizofrenia e eventos psicóticos em geral, mas a partir da observação clínica, nos anos 1950, acerca dos efeitos benéficos da clorpromazina em controlar os sintomas positivos de psicose na esquizofrenia e mania, sabe-se que a hiperatividade da dopamina tem um papel central nesse mecanismo, uma vez que a clorpromazina é um antagonista do receptor central de dopamina do tipo 2 (D2) (Fantegrossi, Wilson, \& Berquist, 2018).

Este não parece ser, todavia, o único neurotransmissor envolvido, já que se verificou que a clozapina é ainda mais eficiente em controlar não só os sintomas positivos, mas também os negativos de transtornos psicóticos (Sawa \& Snyder, 2003). Foi descoberto que a clozapina possui, além de atividade antidopamínica, a capacidade de bloquear receptores de serotonina, de forma que a associação entre psicose e hiperatividade serotoninérgica foi observada pela primeira vez (Sawa \& Snyder, 2003).

Posteriormente, o glutamato também foi associado com sintomas psicóticos, particularmente o receptor central de glutamato do tipo N-metil-D-aspartato (NMDA), entretanto, ao contrário da dopamina e serotonina, o bloqueio deste tipo de receptor, e não sua hiperativação, induz os eventos psicóticos (Fantegrossi et al., 2018).

O estudo neurobiológico de substâncias psicoativas, principalmente drogas ilícitas, estabeleceu uma relação dessas vias com a psicose (Dos Santos, Bouso, \& Hallak, 2017; Ham et al., 2017; Fantegrossi et al., 2018). Os princípios ativos da cannabis, particularmente o tetrahidrocanabidiol (THC), interagem com os sistemas dopaminérgico, serotoninérgico e glutamatérgico, mediados por sua interação com os Receptores Endocanabinoides do Tipo 1 (CB1), induzindo hiperatividade da dopamina e da serotonina e bloqueio dos receptores NMDA (Fantegrossi et al., 2018; Sawa \& Snyder, 2013), fato que é tido como explicação para a relação já bem estabelecida entre esta substância, a esquizofrenia e eventos psicóticos em geral (Murrie et al., 2020; Björkenstam et al., 2013). Além disso, existem evidências de atividade neuronal semelhante em doenças psicóticas e em indivíduos expostos a THC colhidas por exame de imagem funcional (Winton-Brown et al., 2011).

Os principais alucinógenos utilizados, incluindo os naturais (psilocibina, mescalina e ayahuasca) e os sintéticos (LSD e DMT isolada) têm em comum o princípio ativo mais importante: a dimetiltriptamina, que é um poderoso agonista serotoninérgico, o que parece explicar os efeitos semelhantes à psicose durante a intoxicação e também os casos em que o uso induz transtornos psicóticos crônicos (Dos Santos et al., 2017). Semelhante ao que se observou com a cannabis, Müller et al. 
(2018) evidenciaram em um ensaio clínico controlado que indivíduos expostos a doses recreativas de LSD tiveram padrões de ativação neuronal semelhante aos de indivíduos esquizofrênicos.

A cocaína, por sua vez, aumenta a disponibilidade central de dopamina por meio do bloqueio da recaptação présináptica deste neurotransmissor. O mesmo mecanismo ocorre com a serotonina, apesar de ocorrer em menor intensidade (Tang, Martin, \& Cotes, 2014). Para as substâncias anfetamínicas, incluindo a metanfetamina, a liberação excessiva de dopamina parece também ter um papel central na indução de eventos psicóticos (Bramness \& Rognli, 2016; Glasner-Edwards \& Mooney, 2014), mas também já se postulou a participação da serotonina (Glasner-Edwards \& Mooney, 2014), bem como um possível papel de alterações da atividade de vias gabaérgicas (Bramness \& Rognli, 2016).

O álcool possui vias bastante heterogêneas para causa de psicose (Jordaan \& Emsley, 2013). Níveis elevados de betacarbonilas e degeneração do córtex auditivo já foram associados à psicose induzida por álcool (Stankewicz, Richards, \& Salen, 2020), além disso, anormalidades perfusionais observadas por meio de neuroimagem funcional também parecem estar relacionadas, mais especificamente com as alucinações (Stankewicz et al., 2020). No que se refere aos neurotransmissores, sabe-se que a ingestão de álcool aumenta a atividade dopamínica nas vias nigroestratal e mesolímbica (Jordaan \& Emsley, 2013), sendo que esta última é de suma importância na ocorrência de sintomas positivos na esquizofrenia (Fantegrossi et al., 2018).

O diagnóstico de TPIS pode ser dado, segundo a quinta edição do Manual Diagnóstico e Estatístico de Transtornos Mentais (DSM-V), apenas com dados clínicos, tendo como principais critérios a presença de delírio e/ou alucinações, bem como a relação temporal com o uso ou abstinência da substância psicoativa em questão (APA, 2013). Todavia, há uma diferença clínica, por vezes difícil de ser percebida, entre o que são fenômenos esperados durante a intoxicação e o que é um TPIS (APA, 2013). De um modo geral, pode-se dizer que se os sintomas persistirem por longo período após a intoxicação e se forem muito intensos, provavelmente se trata de um TPIS verdadeiro (Tang et al., 2014; Bramness \& Rognli, 2016; Dos Santos et al., 2017; Kurtom et al., 2019; Pearson \& Berry, 2019).

Neste estudo os principais sintomas observados nos pacientes foram delírios paranoides ou persecutórios, delírios místicos, de ressuscitação e delírios de referência. Além disso, os estudos relataram como sintomas vivências alucinatórias (auditivas, visuais, cinestésicas e cenestésicas). A agressividade e/ou agitação psicomotora também foram citadas como sintomas. A sintomatologia produzida no TPIS relacionado à cannabis, cocaína, anfetaminas, alucinógenos ou álcool não diferem de maneira significativa entre si (Ham et al., 2017; Mancheno et al., 2016). Os principais sintomas das principais drogas recreativas estão apresentados na Figura 3. Nas psicoses associadas à cannabis, os sintomas psiquiátricos positivos são os mais frequentes (Hindley et al., 2020), mas há também uma considerável ocorrência de sintomas negativos, como embotamento afetivo, e sintomas psiquiátricos gerais (Hindley et al., 2020), dentre estes, a ansiedade aparece muito frequentemente (Gerlach et al., 2019). Nos quadros causados por alucinógenos, há também uma elevada incidência de sintomas positivos, com alucinações vívidas (Dos Santos et al., 2017) e atividade psicomotora aumentada (Colizzi, Ruggeri, \& Bhattacharyya, 2020), sintomas negativos também compõe o quadro, com destaque para a capacidade de socialização diminuída (Colizzi et al., 2020). 
Figura 3. Principais sintomas psiquiátricos associados a cannabis, alucinógenos, cocaína, anfetaminas e álcool.
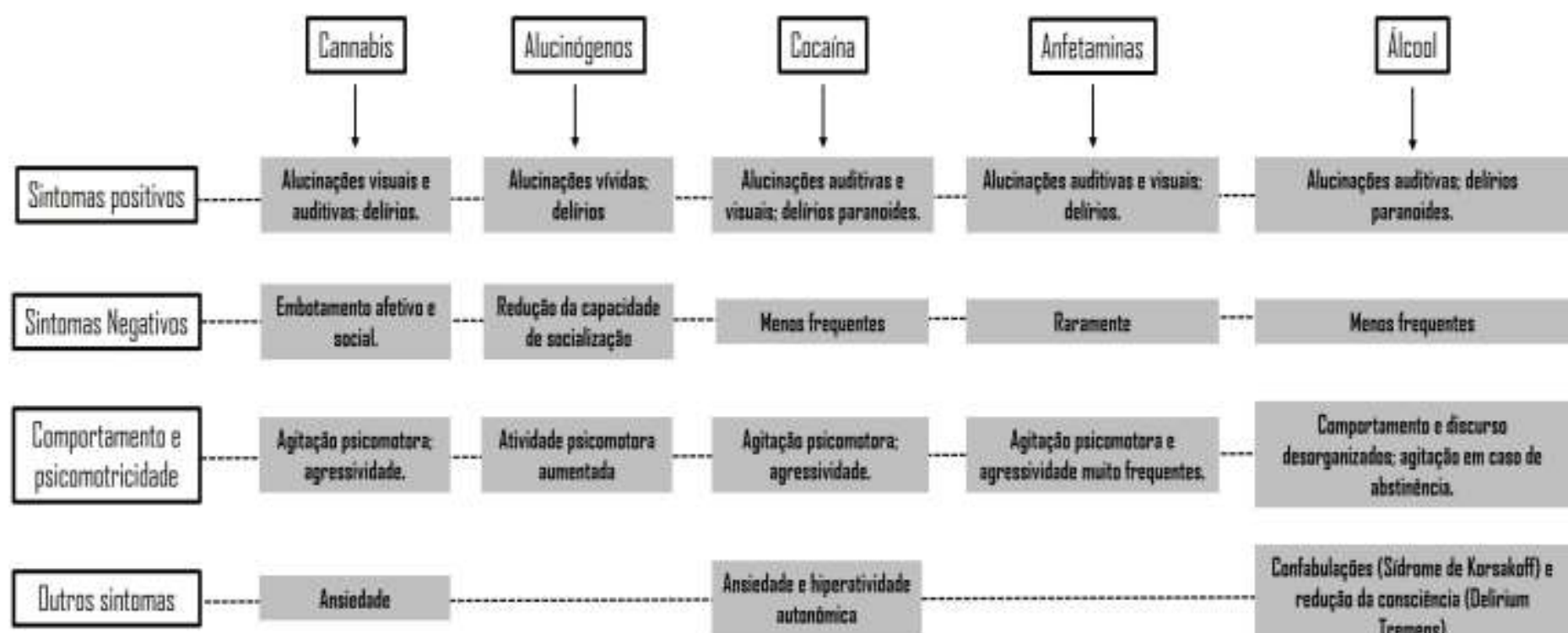

Canfabulaçües (Sidrame de Korsakaff) e redução da conscięacia (Delirium Tremens)

Fonte: Autores (2021).

Nos casos associados à cocaína há um claro predomínio de sintomas positivos, com alucinações auditivas, visuais e delírios de conteúdo paranoide como os fenômenos mais frequentes (Tang et al., 2014). As anfetaminas também estão mais associadas com sintomas positivos (Bramness \& Rognli, 2016), mas têm a particularidade de induzir psicomotricidade aumentada e agitação com quase a mesma frequência (Bramness \& Rognli, 2016), além de não ser tão comum a apresentação com sintomas negativos, principalmente comprometimento da interatividade social (Ham et al., 2017).

Para os casos associados ao álcool, a caracterização diagnóstica é um pouco mais complexa, já que existem não só um, mas três transtornos com sintomatologia psicótica ou semelhante relacionados com esta substância: delirium tremens, síndrome de korsakoff e o transtorno psicótico induzido por álcool (APA, 2013). O delirium tremens é o único entre os três em que há alteração do nível de consciência, além disso é causado por abstinência e não por intoxicação por álcool (Jordaan \& Emsley, 2013). A síndrome de korsakoff é causada pela carência crônica de vitamina B12, o que quase sempre tem relação com alcoolismo crônico, e têm como característica clínica principal as confabulações, que são construções fantasiosas de falsas memórias (APA, 2013). Por conta disso pode haver dificuldade em diferenciar essas construções fantasiosas de ideias delirantes, mas as confabulações têm conteúdos aleatórios, além de variarem quase toda vez em que o paciente é questionado sobre as supostas memórias novamente, diferentemente de um delírio (APA, 2013). O TPIS verdadeiro causado por álcool é caracterizado por sintomas positivos, especialmente alucinações auditivas e delírios paranoides, com a ressalva de que não há alteração no nível de consciência (Jordaan \& Emsley, 2013).

Ainda que a sintomatologia não seja suficiente para que o profissional de saúde saiba exatamente qual é a droga relacionada (APA, 2013), a clínica pode auxiliar no sentido de saber qual será a abordagem inicial (Glasner-Edwards \& Mooney, 2014). Pacientes com sintomas psicóticos causados por álcool ou cocaína frequentemente requerem uso de benzodiazepínicos (Grover \& Ghosh, 2018; Tang et al., 2014), o que não necessariamente se repete nos quadros induzidos por alucinógenos (Neyra-Ontaneda, 2017). Devido ao fato da clínica ser muito semelhante entre as diferentes etiologias de TPIS, a anamnese do paciente, caso este esteja habilitado a responder as perguntas, ou do acompanhante é importante para definir qual a droga indutora (Fiorentini et al., 2011). O exame de urina para pesquisa de metabólitos das principais drogas recreativas têm benefícios nesse sentido, visto que já foi relatado que em cerca de metade dos pacientes o exame dos metabólitos e os relatos 
colhidos podem não corresponder (Fielitz et al., 2010), muito embora a abordagem terapêutica inicial não dependa necessariamente de saber a substância indutora (Fiorentini et al., 2011).

O principal método terapêutico frente a um TPIS é a abstinência e suporte clínico (Fiorentiniet al., 2011), visto que esses distúrbios normalmente são auto-limitados (APA, 2013). Todavia, a necessidade de terapia farmacológica é frequente, seja pelos sintomas de abstinência, com uso de benzodiazepínicos, seja pelos sintomas psicóticos propriamente ditos, com uso de neurolépticos (Glasner-Edwards \& Mooney, 2014; Tang et al., 2014; Kurtom et al., 2019; Neyra-Ontaneda, 2017; Stankewicz et al., 2020; Gerlach et al., 2019). Além disso, especialmente para os quadros causados por cannabis, anfetaminas e alucinógenos, é importante que haja assistência psiquiátrica e psicológica continuada depois que o quadro for resolvido, já que há evidências de que esse tipo de abordagem nesses pacientes diminui consideravelmente o risco de nova internação e de cronificação da psicose (Murrie et al., 2020).

\section{Considerações Finais}

Transtornos psicóticos induzidos por drogas recreativas são distúrbios psiquiátricos graves e com grande potencial de morbidade e limitação funcional. Por conta do crescente aumento do uso destas substâncias no Brasil e no mundo, é possível que sua frequência se torne cada vez maior, bem como as internações psiquiátricas e incapacitação de indivíduos em idades produtiva, de modo que deveria ser considerado entre um dos grandes problemas relacionados às drogas, junto com outros crimes e com a violência. Dessa forma, é fundamental que os profissionais de saúde compreendam que o uso de drogas recreativas pode levar ao desenvolvimento de psicose e saibam avaliar a partir dos principais sintomas e decidir a melhor forma de abordar esses pacientes para que possam oferecer o melhor suporte possível.

\section{Referências}

Albobali, Y., \& Madi, M. Y. (2019). Polysubstance Use Disorder After Sleeve Gastrectomy. Cureus, 11(4), e4388.

American Psychiatric Association. (2013). Transtornos relacionados a substâncias. Em Manual diagnóstico e estatístico de transtornos mentais (5ª ed.). Whashington DC., Estados Unidos da América.

Barrio, P., Gaskell, M., Goti, J., Vilardell, S., \& Fàbregas, J. (2016). Síntomas psicóticos persistentes después del uso abusivo prolongado de mefedrona: una serie de dos casos. Adicciones, 28(3), 154-157.

Björkenstam, E., Björkenstam, C., Hjern, A., Reutfors, J., \& Bodén, R. (2013). A five year diagnostic follow-up of 1,840 patients after a first episode nonschizophrenia and non-affective psychosis. Schizophrenia research, 150(1), 205-210.

Bramness, J. G., \& Rognli, E. B. (2016). Psychosis induced by amphetamines. Current opinion in psychiatry, 29(4), $236-241$.

Carlini, E. A., Galduróz, J. C., Silva, A. M., Noto, A. R., Fonseca, A. M., Carlini, C. M., Oliveira, L. G. et al. (2006). Principais resultados gerais do Brasil 2005. Em II Levantamento domiciliar sobre o uso de drogas psicotrópicas no Brasil: estudo envolvendo as 108 maiores cidades do país. São Paulo, Brasil.

Chan, W. L., Wood, D. M., Hudson, S., \& Dargan, P. I. (2013). Acute psychosis associated with recreational use of benzofuran 6-(2-aminopropyl) benzofuran (6-APB) and cannabis. Journal of medical toxicology: official journal of the American College of Medical Toxicology, 9(3), 278-281.

Chin, J., Forzani, B., Chowdhury, N., Lombardo, S., Rizzo, J. R., \& Ragucci, M. (2015). Rehabilitation essential in the recovery of multifactorial subacute combined degeneration. Annals of physical and rehabilitation medicine, 58(3), 190-192.

Colizzi, M., Ruggeri, M., \& Bhattacharyya, S. (2020). Unraveling the Intoxicating and Therapeutic Effects of Cannabis Ingredients on Psychosis and Cognition. Frontiers in psychology, 11, 833 .

Dannatt, L., Cloete, K. J., Kidd, M., \& Weich, L. (2014). Frequency and correlates of comorbid psychiatric illness in patients with heroin use disorder admitted to Stikland Opioid Detoxification Unit. South Africa. South African Journal of Psychiatry, 20(3), 77-82.

Dos Santos, R. G., Bouso, J. C., \& Hallak, J. (2017). Ayahuasca, dimethyltryptamine, and psychosis: a systematic review of human studies. Therapeutic advances in psychopharmacology, 7(4), 141-157.

Fantegrossi, W. E., Wilson, C. D., \& Berquist, M. D. (2018). Pro-psychotic effects of synthetic cannabinoids: interactions with central dopamine, serotonin, and glutamate systems. Drug metabolism reviews, 50(1), 65-73. 
Fielitz, P., Suárez, H., Escobal, M., Frontini, M. A., López-Rega, G., Navarro, V., Rodríguez-Formoso, L., Storch, A., García, S., \& Campo, O. (2010). Consumo de sustancias psicoactivas en pacientes con trastornos psicóticos ingresados en Sala de Emergencia psiquiátrica: Hospital Vilardebó. Montevideo. Uruguay. Revista chilena de neuro-psiquiatría, 48(2), 106-113.

Filho, F. P., \& Silveira, L. C. (2014). Psicose e drogadição: a construção do caso na clínica de enfermagem. SMAD. Revista eletrônica saúde mental álcool e drogas, 10(1), 29-34.

Fiorentini, A., Volonteri, L. S., Dragogna, F., Rovera, C., Maffini, M., Mauri, M. C., \& Altamura, C. A. (2011). Substance-induced psychoses: a critical review of the literature. Current drug abuse reviews, 4(4), 228-240.

Fontanella, B. J. B. (2010). Sintomas psicóticos e cognitivos associados à busca de tratamento por dependentes de substâncias: um estudo qualitativo. Jornal Brasileiro de Psiquiatria, 59(2), 139-145.

Fullajtar, M., \& Ferencz, C. (2012). Dizájner drog indukálta pszichózis. Neuropsychopharmacologia Hungarica: a Magyar Pszichofarmakologiai Egyesulet lapja, 14(2), 137-140.

Fundação Oswaldo Cruz, \& Instituto de Comunicação e Informação Científica e Tecnológica em Saúde. (2017). Características gerais da população de pesquisa. Em III Levantamento Nacional sobre o uso de drogas pela população brasileira. Rio de Janeiro, Brasil.

Gerlach, J., Koret, B., Gereš, N., Matić, K., Prskalo-Čule, D., Zadravec Vrbanc, T., Lovretić, V., Skopljak, K., Matoš, T., Šimunović Filipčić, I., \& Filipčić, I. (2019). Clinical Challenges in Patients with First Episode Psychosis and Cannabis Use: Mini-Review and a Case Study. Psychiatria Danubina, 31(suppl 2), $162-170$.

Glasner-Edwards, S., \& Mooney, L. J. (2014). Methamphetamine psychosis: epidemiology and management. CNS drugs, 28(12), 1115-1126.

Grover, S., \& Ghosh, A. (2018). Delirium Tremens: Assessment and Management. Journal of clinical and experimental hepatology, 8(4), 460-470.

Guimarães, P. M., Passos, S. R., Calvet, G. A., Hökerberg, Y. H., Lessa, J. L., \& Andrade, C. A. (2014). Suicide risk and alcohol and drug abuse in outpatients with HIV infection and Chagas disease. Brazilian Journal of Psychiatry, 36(2), 131-137.

Ham, S., Kim, T. K., Chung, S., \& Im, H. I. (2017). Drug Abuse and Psychosis: New Insights into Drug-induced Psychosis. Experimental neurobiology, 26(1), $11-24$.

Hindley, G., Beck, K., Borgan, F., Ginestet, C. E., McCutcheon, R., Kleinloog, D., Ganesh, S., Radhakrishnan, R., D'Souza, D. C., \& Howes, O. D. (2020). Psychiatric symptoms caused by cannabis constituents: a systematic review and meta-analysis. The lancet. Psychiatry, 7(4), 344-353.

Ikawa, H., Kanata, S., Akahane, A., Tochigi, M., Hayashi, N., \& Ikebuchi, E. (2019). A case of methamphetamine use disorder presenting a condition of ultrarapid cycler bipolar disorder. SAGE open medical case reports, 7, 2050313X19827739.

Jordaan, G. P., \& Emsley, R. (2014). Alcohol-induced psychotic disorder: a review. Metabolic brain disease, 29(2), $231-243$.

Karila, L., Petit, A., Phan, O., \& Reynaud, M. (2010). Les troubles psychotiques induits par la cocaïne: une revue synthétique. Revue medicale de Liege, 65(11), 623-627.

Kilciksiz, C. M., Keefe, R., Benoit, J., Öngür, D., \& Torous, J. (2020). Verbal memory measurement towards digital perspectives in first-episode psychosis: A review. Schizophrenia Research: Cognition, 21, 100177.

Kurtom, M., Henning, A., \& Espiridion, E. D. (2019). Hallucinogen-persisting Perception Disorder in a 21-year-old Man. Cureus, $11(2)$, e4077.

Mancheno, J. M., Orellana, M. R., Muñoz J. F., Rodas, L. M., Reyes, K. A., Almeida, A. G., \& Vázquez P. A. (2019). Prevalencia de patología dual en el centro de reposo y adicciones (CRA). Cuenca, abril diciembre 2016. Revista de la faculdad de ciencias médicas de la universidad de cuenca, 37(2), 31-39.

Mancke, F., Kaklauskaite, G., Kollmer, J., \& Weiler, M. (2016). Psychiatric comorbidities in a young man with subacute myelopathy induced by abusive nitrous oxide consumption: a case report. Substance abuse and rehabilitation, 7, 155-159.

Marconi, A., Di Forti, M., Lewis, C. M., Murray, R. M., \& Vassos, E. (2016). Meta-analysis of the Association Between the Level of Cannabis Use and Risk of Psychosis. Schizophrenia bulletin, 42(5), 1262-1269.

Müller, F., Dolder, P. C., Schmidt, A., Liechti, M. E., \& Borgwardt, S. (2018). Altered network hub connectivity after acute LSD administration. NeuroImage. Clinical, 18, 694-701.

Murrie, B., Lappin, J., Large, M., \& Sara, G. (2020). Transition of Substance-Induced, Brief, and Atypical Psychoses to Schizophrenia: A Systematic Review and Meta-analysis. Schizophrenia bulletin, 46(3), 505-516.

Neyra-Ontaneda, Diego. (2017). Psicosis inducida por ayahuasca: reporte de un caso. Revista de Neuro-Psiquiatría, 80(4), $265-272$.

Palma-Álvarez, R. F., Ros-Cucurull, E., Ramos-Quiroga, J. A., Roncero, C., \& Grau-López, L. (2019). Cocaine-Induced Psychosis and Asenapine as Treatment: A Case Study. Psychopharmacology bulletin, 49(1), 92-97.

Pearson, N. T., \& Berry, J. H. (2019). Cannabis and Psychosis Through the Lens of DSM-5. International journal of environmental research and public health, 16(21), 4149.

Pereira, A. S., Shitsuka, D. M., Parreira, F. J., \& Shitsuka, R. (2018). Metodologia da pesquisa científica [recurso eletrônico] - 1. ed. - Santa Maria, RS: UFSM, NTE 
Research, Society and Development, v. 10, n. 2, e21910212459, 2021

(CC BY 4.0) | ISSN 2525-3409 | DOI: http://dx.doi.org/10.33448/rsd-v10i2.12459

Personne, M., Westerbergh, J., \& Hammer-Pettersen, L. (2014). »Spice« - syntetiska kannabinoider med riskabla effekter - Fall av akut njursvikt och cerebral ischemi med stroke har rapporterats. Lakartidningen, 111(47), 2105-2107.

Redes, L., Brescia, M.S., Wschebor, M., \& Gutiérrez, M. (2013). Acerca de un caso clínico de patologia dual de psicosis y consumo de sustancias. Revista Psiquiatría del Uruguay, 77(1), 68-74.

Revilla-Zúñiga, J., Rivera-Encinas, M. T., \& Cruzado, L. (2016). Diagnóstico erróneo de psicosis inducida por cocaína en una persona con esquizofrenia y masticadora de hojas de coca. Revista de Neuro-Psiquiatría, 79(2), 119-126.

Roncero, C., Ros-Cucurull, E., Daigre, C., \& Casas, M. (2012). Prevalence and risk factors of psychotic symptoms in cocaine-dependent patients. Actas espanolas de psiquiatria, 40(4), 187-197.

Rounsaville B. J. (2007). DSM-V research agenda: substance abuse/psychosis comorbidity. Schizophrenia bulletin, 33(4), 947-952.

Ryan, J. E., Smeltzer, S. C., \& Sharts-Hopko, N. C. (2019). Challenges to studying illicit drug users. Journal of nursing scholarship, 51(4), 480-488

Sawa, A., \& Snyder, S. H. (2003). Schizophrenia: neural mechanisms for novel therapies. Molecular medicine (Cambridge, Mass.), 9(1-2), 3-9.

Silva, C.C, Savian, C.M, Prevedello, B.P, Zamberlan, C., Dalpian, D.M, Santos, B.Z (2018). Acesso e utilização de serviços odontológicos por gestantes: Revisão integrativa de literatura. Ciência \& Saúde Coletiva, 25(3), 827-835.

Stankewicz, H. A., Richards, J. R., \& Salen, P. (2020). Alcohol Relates Psychosis. In: StatPearls publishing.

Tampi, R. R., Young, J., Hoq, R., Resnick, K., \& Tampi, D. J. (2019). Psychotic disorders in late life: a narrative review. Therapeutic advances in psychopharmacology, 9, 2045125319882798.

Tang, Y., Martin, N. L., \& Cotes, R. O. (2014). Cocaine-induced psychotic disorders: presentation, mechanism, and management. Journal of dual diagnosis, 10(2), 98-105.

Thirthalli, J., \& Benegal, V. (2006). Psychosis among substance users. Current opinion in psychiatry, 19(3), 239-245.

Thomas, E., Lategan, H., Verster, C., Kidd, M., \& Weich, L. (2016). Methamphetamine-induced psychosis: Clinical features, treatment modalities and outcomes. The South African journal of psychiatry: SAJP: the journal of the Society of Psychiatrists of South Africa, 22(1), 980.

Tucker, M. G., Kekulawala, S., Kent, M., Mostafa, S., \& Harvey, R. (2016). Polysubstance-induced relapse of schizoaffective disorder refractory to high-dose antipsychotic medications: a case report. Journal of medical case reports, 10(1), 242.

Ul Haq, E., Shafiq, A., Khan, A. A., Awan, A. A., Ezad, S., Minteer, W. J., \& Omar, B. (2017). "Spice" (Synthetic Marijuana) Induced Acute Myocardial Infarction: A Case Series. Case reports in cardiology, 2017, 9252463.

United Nations Office of Drug and Crime. (2019). Global overview of drug demand and supply. Em World drug report 2019. Viena, Áustria.

Winton-Brown, T. T., Allen, P., Bhattacharyya, S., Borgwardt, S. J., Fusar-Poli, P., Crippa, J. A., Seal, M. L., Martin-Santos, R., Ffytche, D., Zuardi, A. W., Atakan, Z., \& McGuire, P. K. (2011). Modulation of auditory and visual processing by delta-9-tetrahydrocannabinol and cannabidiol: an FMRI study. Neuropsychopharmacology: official publication of the American College of Neuropsychopharmacology, 36(7), 1340-1348.

Zamora-Rodríguez, F. J., Sánchez-Walsen-Hernández, M. R., Guisado-Macias, J. A., \& Vaz-Leal, F. J. (2018). Uso de sustancias y curso del trastorno bipolar en una muestra de pacientes hospitalizados. Actas españolas de psiquiatría, 46(5), 183-192.

Zhao, B., Zhao, L., Li, Z., \& Zhao, R. (2020). Subacute combined degeneration induced by nitrous oxide inhalation: Two case reports. Medicine, 99(18), e19926. 